Acta Crystallographica Section E

Structure Reports

Online

ISSN 1600-5368

\section{Poly[bis $\left(\mu_{4}\right.$-acetato- $\left.\kappa^{4} O: O: O^{\prime}: O^{\prime}\right) \operatorname{bis}\left(\mu_{3}-\right.$ acetato- $\left.\kappa^{3} O: O: O\right)\left(\mu_{2}\right.$-acetato- $\left.\kappa^{2} O: O^{\prime}\right)$ - $\left(\mu_{2}\right.$-acetic acid- $\left.\kappa^{2} O: O^{\prime}\right)$ di- $\mu$-aqua- copper(II)trisodium]}

\author{
Xiang-Yu Jiang and Xi-Gui Yue* \\ Alan G. MacDiarmid Institute, Jilin University, Changchun 130012, People's \\ Republic of China \\ Correspondence e-mail: yuexigui@jlu.edu.cn
}

Received 19 August 2010; accepted 6 September 2010

Key indicators: single-crystal X-ray study; $T=290 \mathrm{~K}$; mean $\sigma(\mathrm{C}-\mathrm{C})=0.003 \AA$; $R$ factor $=0.023 ; w R$ factor $=0.068 ;$ data-to-parameter ratio $=17.6$.

In the title compound, $\left[\mathrm{CuNa}_{3}\left(\mathrm{CH}_{3} \mathrm{CO}_{2}\right)_{5}\left(\mathrm{CH}_{3} \mathrm{COOH}\right)\right.$ $\left.\left(\mathrm{H}_{2} \mathrm{O}\right)_{2}\right]_{n}$, the $\mathrm{Cu}^{\mathrm{II}}$ atom lies on an inversion center and is coordinated by four $\mathrm{O}$ atoms from four acetate ligands, leading to a square-planar geometry. One $\mathrm{Na}^{\mathrm{I}}$ atom, lying on an inversion center, is coordinated by four $\mathrm{O}$ atoms from four acetate ligands and two bridging water molecules in a distorted octahedral geometry. The other $\mathrm{Na}^{\mathrm{I}}$ atom is coordinated by five $\mathrm{O}$ atoms from five acetate ligands and a bridging water molecule. A hydroxy $\mathrm{H}$ atom lies on a twofold rotation axis and is shared by two acetate ligands. The crystal packing exhibits a polymeric layer parallel to (100), which is further stablized by intralayer $\mathrm{O}-\mathrm{H} \cdots \mathrm{O}$ hydrogen bonds. The layers are linked by interlayer $\mathrm{O}-\mathrm{H} \cdots \mathrm{O}$ hydrogen bonds.

\section{Related literature}

For related structures, see: Chiari et al. (1988); Vives et al. (2003).

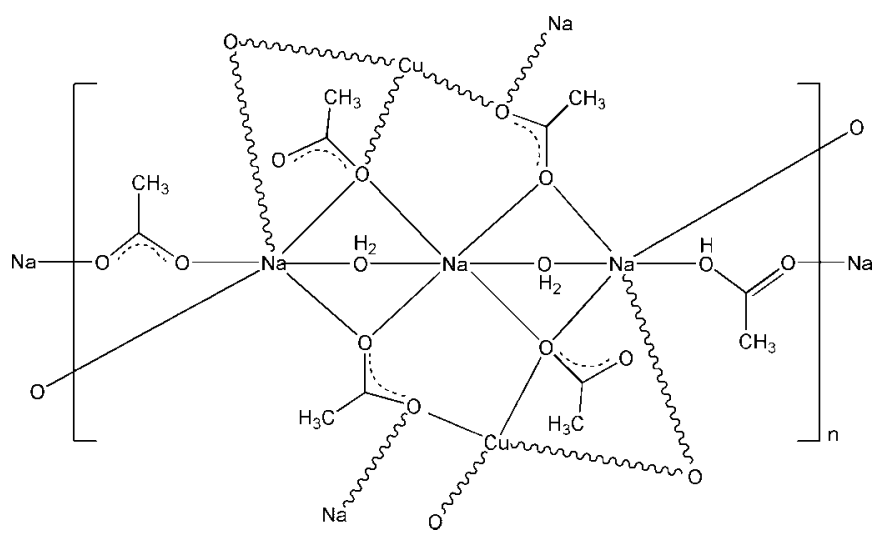

\section{Experimental}

Crystal data

$\left[\mathrm{CuNa}_{3}\left(\mathrm{C}_{2} \mathrm{H}_{3} \mathrm{O}_{2}\right)_{5}\left(\mathrm{C}_{2} \mathrm{H}_{4} \mathrm{O}_{2}\right)\left(\mathrm{H}_{2} \mathrm{O}\right)_{2}\right]$

$M_{r}=523.81$

Monoclinic, $C 2 / c$

$a=14.571$ (6) А

$b=6.768(3) \AA$

$c=22.653(10) \AA$

$\beta=103.426(19)^{\circ}$

$V=2173.0(16) \AA^{3}$

$Z=4$

Mo $K \alpha$ radiation

$\mu=1.13 \mathrm{~mm}^{-1}$

$T=290 \mathrm{~K}$

$0.27 \times 0.25 \times 0.23 \mathrm{~mm}$

\section{Data collection}

Rigaku R-AXIS RAPID diffractometer

Absorption correction: multi-scan (ABSCOR; Higashi, 1995)

$$
T_{\min }=0.752, T_{\max }=0.777
$$

\section{Refinement}

$R\left[F^{2}>2 \sigma\left(F^{2}\right)\right]=0.023$

$w R\left(F^{2}\right)=0.068$

$S=1.03$

2493 reflections

10214 measured reflections 2493 independent reflections 2192 reflections with $I>2 \sigma(I)$ $R_{\text {int }}=0.023$

Table 1

Hydrogen-bond geometry $\left(\AA{ }^{\circ}\right)$.

\begin{tabular}{lllll}
\hline$D-\mathrm{H} \cdots A$ & $D-\mathrm{H}$ & $\mathrm{H} \cdots A$ & $D \cdots A$ & $D-\mathrm{H} \cdots A$ \\
\hline O7-H7A $\cdots \mathrm{O} 1$ & 0.79 & 2.05 & $2.835(2)$ & 172 \\
$\mathrm{O}^{\mathrm{O}}-\mathrm{H} 7 B \cdots \mathrm{O} 1^{\mathrm{i}}$ & 0.80 & 2.03 & $2.8104(19)$ & 168
\end{tabular}

Symmetry code: (i) $-x+\frac{1}{2},-y+\frac{3}{2},-z+1$.

Data collection: RAPID-AUTO (Rigaku, 1998); cell refinement: RAPID-AUTO; data reduction: CrystalStructure (Rigaku/MSC, 2002); program(s) used to solve structure: SHELXS97 (Sheldrick, 2008); program(s) used to refine structure: SHELXL97 (Sheldrick, 2008); molecular graphics: PLATON (Spek, 2009) and DIAMOND (Brandenburg, 1999); software used to prepare material for publication: SHELXL97.

Supplementary data and figures for this paper are available from the IUCr electronic archives (Reference: HY2345).

\section{References}

Brandenburg, K. (1999). DIAMOND. Crystal Impact GbR, Bonn, Germany. Chiari, B., Piovesana, O., Tarantelli, T. \& Zanazzi, P. F. (1988). Inorg. Chem. 27, $3246-3248$

Higashi, T. (1995). ABSCOR. Rigaku Corporation, Tokyo, Japan.

Rigaku (1998). RAPID-AUTO. Rigaku Corporation, Tokyo, Japan.

Rigaku/MSC (2002). CrystalStructure. Rigaku/MSC Inc., The Woodlands, Texas, USA.

Sheldrick, G. M. (2008). Acta Cryst. A64, 112-122.

Spek, A. L. (2009). Acta Cryst. D65, 148-155.

Vives, G., Mason, S. A., Prince, P. D., Junk, P. C. \& Steed, J. W. (2003). Cryst. Growth Des. 3, 699-704. 


\section{supporting information}

Acta Cryst. (2010). E66, m1244 [doi:10.1107/S1600536810035683]

\section{Poly $\left[\right.$ bis $\left(\mu_{4}\right.$-acetato- $\left.\kappa^{4} O: O: O^{\prime}: O^{\prime}\right)$ bis $\left(\mu_{3}\right.$-acetato- $\left.\kappa^{3} O: O: O\right)\left(\mu_{2}\right.$-acetato- $\left.\kappa^{2} O: O^{\prime}\right)$ $\left(\mu_{2}\right.$-acetic acid- $\left.\kappa^{2} O: O^{\prime}\right)$ di- $\mu$-aquacopper(II)trisodium]}

\section{Xiang-Yu Jiang and Xi-Gui Yue}

\section{S1. Comment}

There has been increasing interest in the study of copper-containing complexes due to their various coordination styles and potential applications. We report here the crystal structure of the title compound, a new copper complex with acetate. In the title compound, as shown in Fig. 1, the $\mathrm{Cu}^{\mathrm{II}}$ atom lies on an inversion center and is four-coordinated by four $\mathrm{O}$ atoms from four acetate ligands, forming a square-planar coordination geometry. In a comparison with the tiltle compound, the complexes previously reported (Chiari et al., 1988; Vives et al., 2003) show different coordination behaviors of the central $\mathrm{Cu}^{\mathrm{II}}$ ion. The two $\mathrm{Na}^{\mathrm{I}}$ ions are each coordinated by six $\mathrm{O}$ atoms, forming a distorted octahedral coordination geometry. Na1 atom lies on an inversion center, while $\mathrm{Na} 2$ atom is on a general position. In the crystal structure, the metal ions, acetate ligands and water molecules are connected each other, forming a two-dimensional network (Fig. 2). The crystal packing is further stabilized by intermolecular $\mathrm{O}-\mathrm{H} \cdots \mathrm{O}$ hydrogen bonds (Table 1).

\section{S2. Experimental}

The title compound was prepared as follows: copper acetate dihydrate $(2.18 \mathrm{~g}, 0.01 \mathrm{~mol})$ was added to a solution of glacial acetic acid $(3 \mathrm{ml})$ in $15 \mathrm{ml}$ water. Then $10 \mathrm{ml} \mathrm{NaOH}(3 \mathrm{~mol} / L)$ was added into the mixture. The mixture was heated and stirred for half an hour and then filtered. The filtrate was allowed to stand at room temperature for several days, giving blue block-shaped crystals.

\section{S3. Refinement}

C-bound $\mathrm{H}$ atoms were placed in calculated positions and refined as riding atoms, with $\mathrm{C}-\mathrm{H}=0.96 \AA$ and with $U_{\text {iso }}(\mathrm{H})=$ $1.5 U_{\text {eq }}(\mathrm{C}) . \mathrm{H}$ atoms of water and carboxyl group are located in a difference Fourier map and refined as riding, with $U_{\text {iso }}(\mathrm{H})=1.5 U_{\text {eq }}(\mathrm{O})$. 


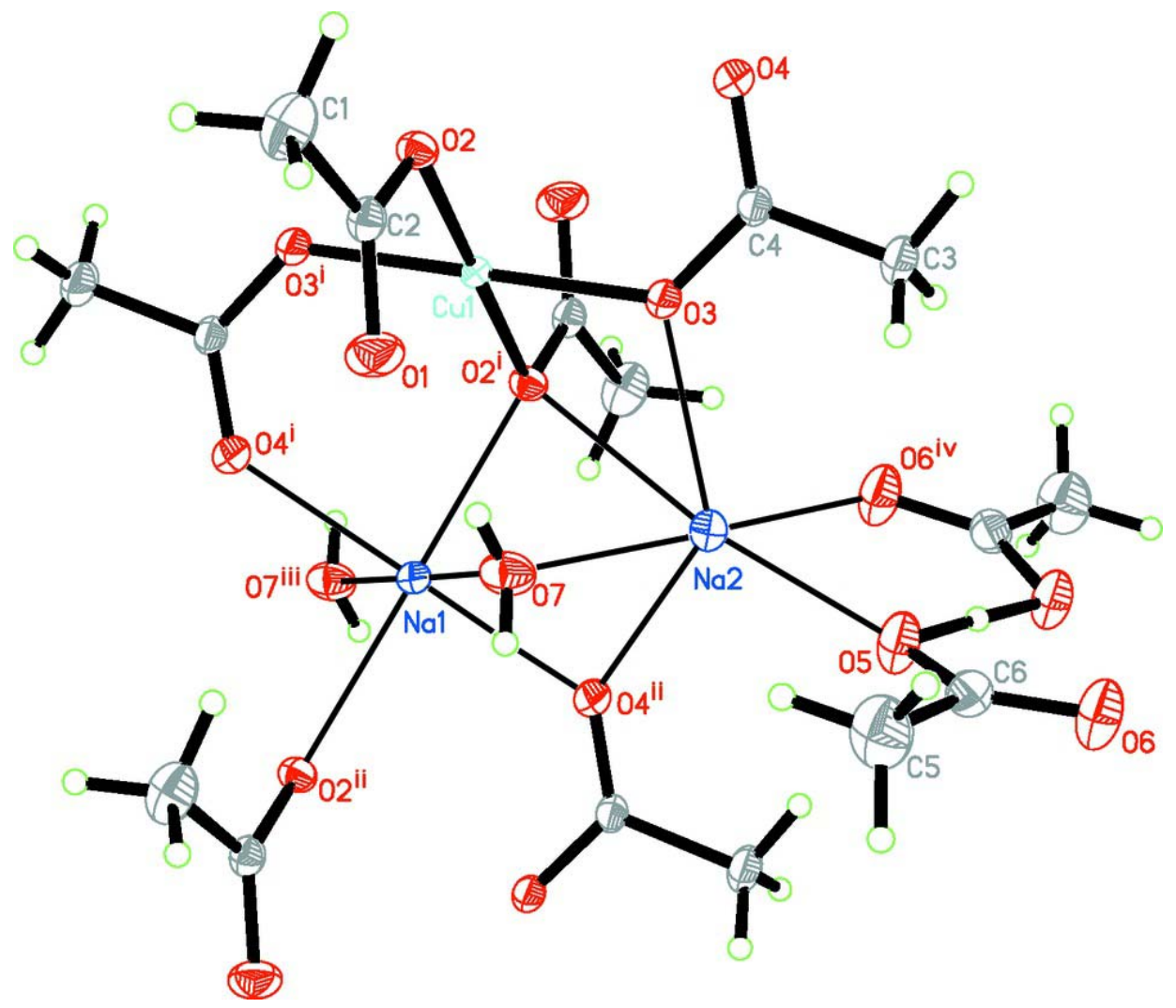

\section{Figure 1}

The structure of the title compound. Displacement ellipsoids are drawn at the $30 \%$ probability level. [Symmetry codes: (i) $-x+1,-y+2,-z+1$; (ii) $x, y-1, z$; (iii) $-x+1,-y+1,-z+1$; (iv) $-x+1, y,-z+1 / 2$.] 


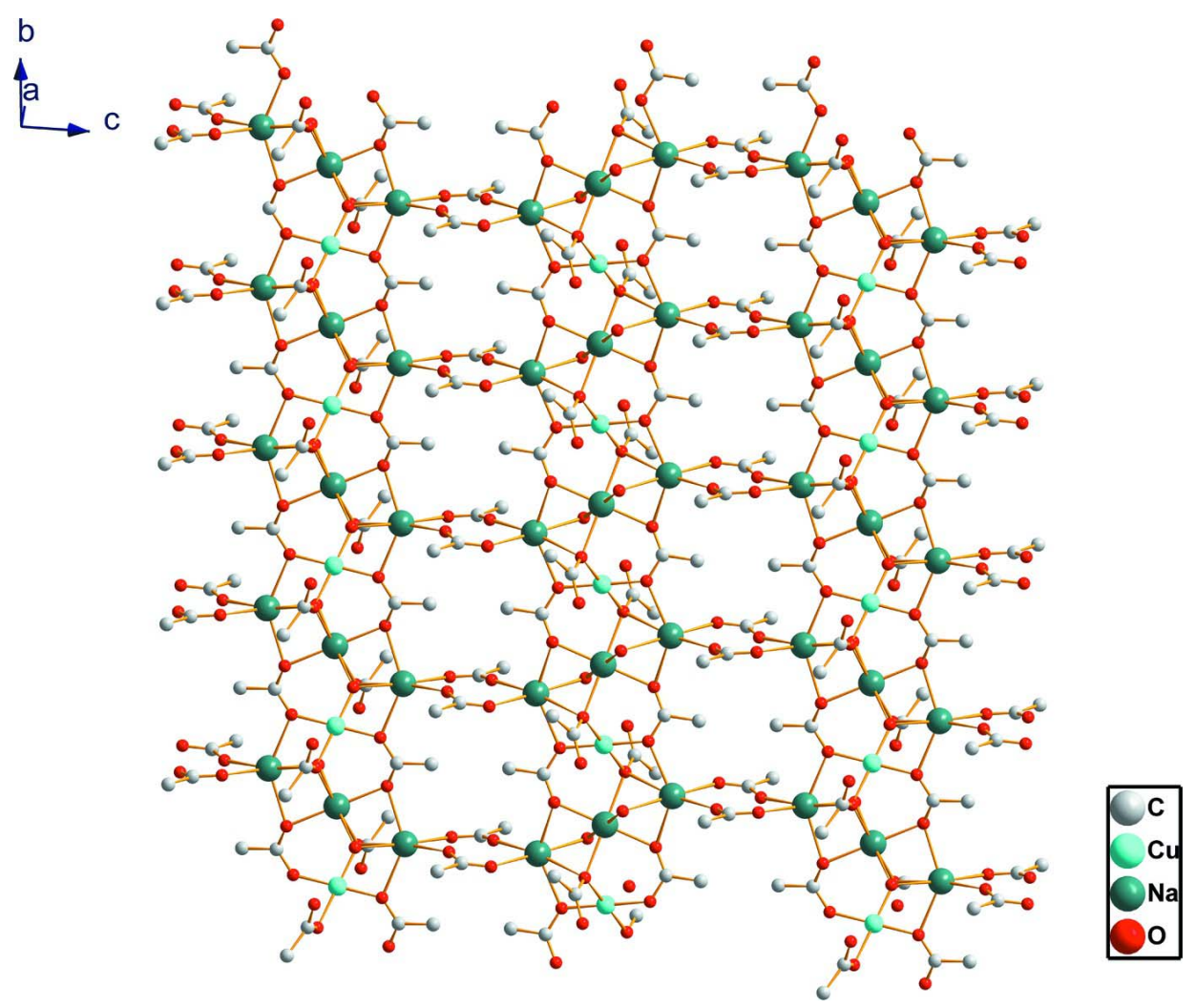

\section{Figure 2}

A view of the two-dimensional structure in the title compound.

Poly[bis $\left(\mu_{4}\right.$-acetato- $\left.\kappa^{4} O: O: O^{\prime}: O^{\prime}\right)$ bis $\left(\mu_{3}\right.$-acetato- $\left.\kappa^{3} O: O: O\right)\left(\mu_{2}\right.$-acetato- $\left.\kappa^{2} O: O^{\prime}\right)\left(\mu_{2}\right.$-acetic acid- $\left.\kappa^{2} O: O^{\prime}\right)$ di- $\mu$ aquacopper(II)trisodium]

Crystal data

$\left[\mathrm{CuNa}_{3}\left(\mathrm{C}_{2} \mathrm{H}_{3} \mathrm{O}_{2}\right)_{5}\left(\mathrm{C}_{2} \mathrm{H}_{4} \mathrm{O}_{2}\right)\left(\mathrm{H}_{2} \mathrm{O}\right)_{2}\right]$

$M_{r}=523.81$

Monoclinic, $C 2 / c$

Hall symbol: $-\mathrm{C} 2 \mathrm{yc}$

$a=14.571(6) \AA$

$b=6.768(3) \AA$

$c=22.653(10) \AA$

$\beta=103.426(19)^{\circ}$

$V=2173.0(16) \AA^{3}$

$Z=4$

$F(000)=1076$

$D_{\mathrm{x}}=1.601 \mathrm{Mg} \mathrm{m}^{-3}$

Mo $K \alpha$ radiation, $\lambda=0.71073 \AA$

Cell parameters from 8924 reflections

$\theta=3.0-27.5^{\circ}$

$\mu=1.13 \mathrm{~mm}^{-1}$

$T=290 \mathrm{~K}$

Block, blue

$0.27 \times 0.25 \times 0.23 \mathrm{~mm}$

\section{Data collection}

Rigaku R-AXIS RAPID diffractometer

Radiation source: rotaton anode

Graphite monochromator

$\omega$ scans

Absorption correction: multi-scan

(ABSCOR; Higashi, 1995)

$T_{\min }=0.752, T_{\max }=0.777$

10214 measured reflections

2493 independent reflections

2192 reflections with $I>2 \sigma(I)$

$R_{\text {int }}=0.023$

$\theta_{\max }=27.5^{\circ}, \theta_{\min }=3.0^{\circ}$

$h=-18 \rightarrow 18$

$k=-8 \rightarrow 8$

$l=-29 \rightarrow 29$ 


\section{Refinement}

Refinement on $F^{2}$

Least-squares matrix: full

$R\left[F^{2}>2 \sigma\left(F^{2}\right)\right]=0.023$

$w R\left(F^{2}\right)=0.068$

$S=1.03$

2493 reflections

142 parameters

0 restraints

Primary atom site location: structure-invariant direct methods
Secondary atom site location: difference Fourier map

Hydrogen site location: inferred from neighbouring sites

$\mathrm{H}$-atom parameters constrained

$w=1 /\left[\sigma^{2}\left(F_{\mathrm{o}}{ }^{2}\right)+(0.039 P)^{2}+1.7139 P\right]$ where $P=\left(F_{\mathrm{o}}{ }^{2}+2 F_{\mathrm{c}}{ }^{2}\right) / 3$

$(\Delta / \sigma)_{\max }=0.011$

$\Delta \rho_{\max }=0.30 \mathrm{e}^{-3}$

$\Delta \rho_{\min }=-0.32$ e $\AA^{-3}$

Special details

Experimental. (See detailed section in the paper)

Fractional atomic coordinates and isotropic or equivalent isotropic displacement parameters $\left(\AA^{2}\right)$

\begin{tabular}{|c|c|c|c|c|}
\hline & $x$ & $y$ & $z$ & $U_{\text {iso }} * / U_{\text {eq }}$ \\
\hline $\mathrm{C} 1$ & $0.26112(13)$ & $1.3050(3)$ & $0.41200(9)$ & $0.0451(5)$ \\
\hline H1A & 0.2567 & 1.2814 & 0.3696 & $0.068^{*}$ \\
\hline H1B & 0.2834 & 1.4370 & 0.4221 & $0.068^{*}$ \\
\hline $\mathrm{H} 1 \mathrm{C}$ & 0.2000 & 1.2893 & 0.4206 & $0.068^{*}$ \\
\hline $\mathrm{C} 2$ & $0.32852(10)$ & $1.1606(2)$ & $0.44894(7)$ & $0.0252(3)$ \\
\hline $\mathrm{C} 3$ & $0.49416(15)$ & $1.1930(3)$ & $0.67768(7)$ & $0.0400(4)$ \\
\hline $\mathrm{H} 3 \mathrm{~A}$ & 0.4943 & 1.3232 & 0.6945 & $0.060 *$ \\
\hline H3B & 0.5462 & 1.1188 & 0.7010 & $0.060^{*}$ \\
\hline $\mathrm{H} 3 \mathrm{C}$ & 0.4361 & 1.1277 & 0.6788 & $0.060 *$ \\
\hline $\mathrm{C} 4$ & $0.50335(11)$ & 1.2077 (2) & $0.61301(6)$ & $0.0237(3)$ \\
\hline $\mathrm{C} 5$ & $0.25974(15)$ & $0.7130(4)$ & $0.69424(11)$ & $0.0614(6)$ \\
\hline $\mathrm{H} 5 \mathrm{~A}$ & 0.2259 & 0.5966 & 0.7010 & $0.092 *$ \\
\hline H5B & 0.2658 & 0.7151 & 0.6529 & $0.092 *$ \\
\hline $\mathrm{H} 5 \mathrm{C}$ & 0.2259 & 0.8282 & 0.7020 & $0.092^{*}$ \\
\hline C6 & 0.35605 (13) & $0.7116(3)$ & $0.73620(8)$ & $0.0352(4)$ \\
\hline $\mathrm{Cu} 1$ & 0.5000 & 1.0000 & 0.5000 & $0.01852(8)$ \\
\hline $\mathrm{Na} 1$ & 0.5000 & 0.5000 & 0.5000 & $0.02776(19)$ \\
\hline $\mathrm{Na} 2$ & $0.50180(5)$ & $0.70788(10)$ & $0.62598(3)$ & $0.03245(16)$ \\
\hline $\mathrm{O} 1$ & $0.30176(9)$ & 0.99791 (19) & $0.46272(7)$ & $0.0430(3)$ \\
\hline $\mathrm{O} 2$ & $0.41529(7)$ & $1.21412(16)$ & $0.46414(5)$ & $0.0244(2)$ \\
\hline $\mathrm{O} 3$ & $0.49001(8)$ & $1.04618(17)$ & $0.58292(5)$ & $0.0282(2)$ \\
\hline $\mathrm{O} 4$ & $0.52322(10)$ & $1.36734(18)$ & $0.59343(5)$ & $0.0374(3)$ \\
\hline O5 & $0.42574(9)$ & $0.6909(2)$ & $0.71094(6)$ & $0.0455(3)$ \\
\hline H5 & 0.5000 & 0.6936 & 0.7500 & $0.068 *$ \\
\hline O6 & $0.36605(10)$ & $0.7272(3)$ & $0.79096(6)$ & $0.0517(4)$ \\
\hline $\mathrm{O} 7$ & $0.37443(8)$ & $0.67127(19)$ & $0.53741(6)$ & $0.0382(3)$ \\
\hline H7B & 0.3291 & 0.6111 & 0.5410 & $0.057^{*}$ \\
\hline H7A & 0.3518 & 0.7657 & 0.5191 & $0.057^{*}$ \\
\hline
\end{tabular}


Atomic displacement parameters $\left(\AA^{2}\right)$

\begin{tabular}{lllllll}
\hline & $U^{11}$ & $U^{22}$ & $U^{33}$ & $U^{12}$ & $U^{13}$ & $U^{23}$ \\
\hline C1 & $0.0326(9)$ & $0.0591(13)$ & $0.0409(10)$ & $0.0154(9)$ & $0.0033(7)$ & $0.0106(9)$ \\
C2 & $0.0234(7)$ & $0.0315(8)$ & $0.0213(7)$ & $0.0002(6)$ & $0.0061(5)$ & $0.0000(6)$ \\
C3 & $0.0630(12)$ & $0.0376(10)$ & $0.0218(8)$ & $-0.0002(9)$ & $0.0148(8)$ & $0.0001(7)$ \\
C4 & $0.0287(7)$ & $0.0242(7)$ & $0.0184(6)$ & $0.0026(6)$ & $0.0058(5)$ & $0.0003(6)$ \\
C5 & $0.0415(11)$ & $0.0837(18)$ & $0.0532(13)$ & $0.0000(11)$ & $-0.0011(10)$ & $-0.0001(12)$ \\
C6 & $0.0386(9)$ & $0.0332(9)$ & $0.0328(8)$ & $-0.0008(7)$ & $0.0063(7)$ & $0.0024(7)$ \\
Cu1 & $0.02176(13)$ & $0.01720(13)$ & $0.01633(12)$ & $-0.00190(9)$ & $0.00390(9)$ & $0.00037(9)$ \\
Na1 & $0.0418(5)$ & $0.0207(4)$ & $0.0213(4)$ & $-0.0071(4)$ & $0.0084(4)$ & $-0.0001(3)$ \\
Na2 & $0.0433(4)$ & $0.0303(3)$ & $0.0235(3)$ & $-0.0038(3)$ & $0.0071(3)$ & $-0.0006(3)$ \\
O1 & $0.0336(6)$ & $0.0445(8)$ & $0.0488(8)$ & $-0.0151(5)$ & $0.0055(6)$ & $0.0105(6)$ \\
O2 & $0.0228(5)$ & $0.0218(5)$ & $0.0271(5)$ & $-0.0013(4)$ & $0.0026(4)$ & $0.0006(4)$ \\
O3 & $0.0426(6)$ & $0.0230(5)$ & $0.0205(5)$ & $-0.0008(5)$ & $0.0102(4)$ & $-0.0001(4)$ \\
O4 & $0.0617(8)$ & $0.0254(6)$ & $0.0243(5)$ & $-0.0066(6)$ & $0.0084(5)$ & $0.0017(5)$ \\
O5 & $0.0401(7)$ & $0.0691(10)$ & $0.0281(6)$ & $-0.0009(7)$ & $0.0095(5)$ & $-0.0013(6)$ \\
O6 & $0.0430(7)$ & $0.0822(12)$ & $0.0316(7)$ & $0.0048(7)$ & $0.0122(6)$ & $0.0004(7)$ \\
O7 & $0.0270(6)$ & $0.0361(7)$ & $0.0502(7)$ & $-0.0062(5)$ & $0.0060(5)$ & $0.0030(6)$ \\
& & & & & & \\
\hline
\end{tabular}

Geometric parameters $\left(\AA,{ }^{\circ}\right)$

\begin{tabular}{|c|c|c|c|}
\hline $\mathrm{C} 1-\mathrm{C} 2$ & $1.496(2)$ & $\mathrm{Cu} 1-\mathrm{O} 3$ & $1.9426(13)$ \\
\hline $\mathrm{C} 1-\mathrm{H} 1 \mathrm{~A}$ & 0.9600 & $\mathrm{Cu} 1-\mathrm{O} 2$ & $1.9540(12)$ \\
\hline $\mathrm{C} 1-\mathrm{H} 1 \mathrm{~B}$ & 0.9600 & $\mathrm{Cu} 1-\mathrm{Na} 1^{\mathrm{i}}$ & $3.3842(14)$ \\
\hline $\mathrm{C} 1-\mathrm{H} 1 \mathrm{C}$ & 0.9600 & $\mathrm{Cu} 1-\mathrm{Na} 2^{\mathrm{ii}}$ & $3.4669(13)$ \\
\hline $\mathrm{C} 2-\mathrm{O} 1$ & $1.232(2)$ & $\mathrm{Na} 1-\mathrm{O} 4^{\mathrm{iii}}$ & $2.2512(14)$ \\
\hline $\mathrm{C} 2-\mathrm{O} 2$ & $1.2831(18)$ & $\mathrm{Na} 1-\mathrm{O} 2^{\mathrm{iii}}$ & $2.3371(13)$ \\
\hline $\mathrm{C} 3-\mathrm{C} 4$ & $1.505(2)$ & $\mathrm{Na} 1-\mathrm{O} 7$ & $2.4759(15)$ \\
\hline $\mathrm{C} 3-\mathrm{H} 3 \mathrm{~A}$ & 0.9600 & $\mathrm{Na} 1-\mathrm{Na} 2^{\mathrm{iv}}$ & $3.1765(13)$ \\
\hline $\mathrm{C} 3-\mathrm{H} 3 \mathrm{~B}$ & 0.9600 & $\mathrm{Na} 2-\mathrm{O}^{\mathrm{v}}$ & $2.3627(18)$ \\
\hline $\mathrm{C} 3-\mathrm{H} 3 \mathrm{C}$ & 0.9600 & $\mathrm{Na} 2-\mathrm{O} 7$ & $2.4088(16)$ \\
\hline $\mathrm{C} 4-\mathrm{O} 4$ & $1.228(2)$ & $\mathrm{Na} 2-\mathrm{O} 5$ & $2.4362(17)$ \\
\hline $\mathrm{C} 4-\mathrm{O} 3$ & $1.2792(19)$ & $\mathrm{Na} 2-\mathrm{O} 4^{\mathrm{iii}}$ & $2.4618(17)$ \\
\hline $\mathrm{C} 5-\mathrm{C} 6$ & $1.501(3)$ & $\mathrm{Na} 2-\mathrm{O} 3$ & $2.4792(16)$ \\
\hline $\mathrm{C} 5-\mathrm{H} 5 \mathrm{~A}$ & 0.9600 & $\mathrm{Na} 2-\mathrm{O} 2^{\mathrm{ii}}$ & $2.6554(15)$ \\
\hline C5-H5B & 0.9600 & $\mathrm{O} 5-\mathrm{H} 5$ & 1.2286 \\
\hline $\mathrm{C} 5-\mathrm{H} 5 \mathrm{C}$ & 0.9600 & $\mathrm{O} 7-\mathrm{H} 7 \mathrm{~B}$ & 0.7965 \\
\hline $\mathrm{C} 6-\mathrm{O} 6$ & $1.220(2)$ & $\mathrm{O} 7-\mathrm{H} 7 \mathrm{~A}$ & 0.7910 \\
\hline $\mathrm{C} 6-\mathrm{O} 5$ & $1.284(2)$ & & \\
\hline $\mathrm{C} 2-\mathrm{C} 1-\mathrm{H} 1 \mathrm{~A}$ & 109.5 & $\mathrm{O} 2^{\mathrm{ii}}-\mathrm{Na} 1-\mathrm{O} 7$ & $82.30(5)$ \\
\hline $\mathrm{C} 2-\mathrm{C} 1-\mathrm{H} 1 \mathrm{~B}$ & 109.5 & 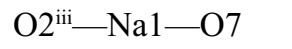 & $97.70(5)$ \\
\hline $\mathrm{H} 1 \mathrm{~A}-\mathrm{C} 1-\mathrm{H} 1 \mathrm{~B}$ & 109.5 & $\mathrm{O} 7^{\mathrm{iv}}-\mathrm{Na} 1-\mathrm{O} 7$ & 180.0 \\
\hline $\mathrm{C} 2-\mathrm{C} 1-\mathrm{H} 1 \mathrm{C}$ & 109.5 & $\mathrm{O} 4^{\mathrm{ii}}-\mathrm{Na} 1-\mathrm{Na} 2^{\mathrm{iv}}$ & $50.50(4)$ \\
\hline $\mathrm{H} 1 \mathrm{~A}-\mathrm{C} 1-\mathrm{H} 1 \mathrm{C}$ & 109.5 & 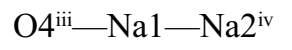 & $129.50(4)$ \\
\hline $\mathrm{H} 1 \mathrm{~B}-\mathrm{C} 1-\mathrm{H} 1 \mathrm{C}$ & 109.5 & $\mathrm{O} 2^{\mathrm{ii}}-\mathrm{Na} 1-\mathrm{Na} 2^{\mathrm{iv}}$ & $124.93(3)$ \\
\hline $\mathrm{O} 1-\mathrm{C} 2-\mathrm{O} 2$ & $122.32(14)$ & 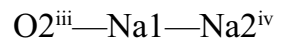 & $55.07(3)$ \\
\hline
\end{tabular}




\begin{tabular}{|c|c|c|c|}
\hline $\mathrm{O} 1-\mathrm{C} 2-\mathrm{C} 1$ & $121.35(15)$ & $\mathrm{O} 7^{\mathrm{iv}}-\mathrm{Na} 1-\mathrm{Na} 2^{\mathrm{iv}}$ & $48.52(3)$ \\
\hline $\mathrm{O} 2-\mathrm{C} 2-\mathrm{C} 1$ & $116.31(15)$ & $\mathrm{O} 7-\mathrm{Na} 1-\mathrm{Na} 2^{\mathrm{iv}}$ & $131.48(4)$ \\
\hline $\mathrm{C} 4-\mathrm{C} 3-\mathrm{H} 3 \mathrm{~A}$ & 109.5 & $\mathrm{O} 4^{\mathrm{ii}}-\mathrm{Na} 1-\mathrm{Cu} 1^{\mathrm{iii}}$ & $113.51(3)$ \\
\hline $\mathrm{C} 4-\mathrm{C} 3-\mathrm{H} 3 \mathrm{~B}$ & 109.5 & 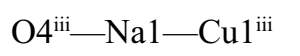 & $66.49(3)$ \\
\hline $\mathrm{H} 3 \mathrm{~A}-\mathrm{C} 3-\mathrm{H} 3 \mathrm{~B}$ & 109.5 & 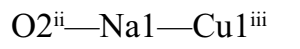 & $145.89(3)$ \\
\hline $\mathrm{C} 4-\mathrm{C} 3-\mathrm{H} 3 \mathrm{C}$ & 109.5 & 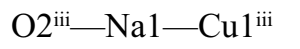 & $34.11(3)$ \\
\hline $\mathrm{H} 3 \mathrm{~A}-\mathrm{C} 3-\mathrm{H} 3 \mathrm{C}$ & 109.5 & $\mathrm{O} 7^{\mathrm{iv}}-\mathrm{Na} 1-\mathrm{Cu} 1^{\mathrm{iii}}$ & $62.08(3)$ \\
\hline $\mathrm{H} 3 \mathrm{~B}-\mathrm{C} 3-\mathrm{H} 3 \mathrm{C}$ & 109.5 & $\mathrm{O} 7-\mathrm{Na} 1-\mathrm{Cu} 1^{\mathrm{iii}}$ & $117.92(3)$ \\
\hline $\mathrm{O} 4-\mathrm{C} 4-\mathrm{O} 3$ & $125.37(14)$ & $\mathrm{Na} 2^{\mathrm{iv}}-\mathrm{Na} 1-\mathrm{Cu} 1^{\mathrm{iii}}$ & $63.708(19)$ \\
\hline $\mathrm{O} 4-\mathrm{C} 4-\mathrm{C} 3$ & $119.44(15)$ & $\mathrm{O}^{\mathrm{v}}-\mathrm{Na} 2-\mathrm{O} 7$ & $175.43(6)$ \\
\hline $\mathrm{O} 3-\mathrm{C} 4-\mathrm{C} 3$ & $115.18(14)$ & $\mathrm{O} 6^{\mathrm{v}}-\mathrm{Na} 2-\mathrm{O} 5$ & $79.03(6)$ \\
\hline $\mathrm{C} 6-\mathrm{C} 5-\mathrm{H} 5 \mathrm{~A}$ & 109.5 & $\mathrm{O} 7-\mathrm{Na} 2-\mathrm{O} 5$ & $104.44(6)$ \\
\hline $\mathrm{C} 6-\mathrm{C} 5-\mathrm{H} 5 \mathrm{~B}$ & 109.5 & $\mathrm{O}^{\mathrm{v}}-\mathrm{Na} 2-\mathrm{O} 4^{\mathrm{iii}}$ & $98.84(6)$ \\
\hline $\mathrm{H} 5 \mathrm{~A}-\mathrm{C} 5-\mathrm{H} 5 \mathrm{~B}$ & 109.5 & $\mathrm{O} 7-\mathrm{Na} 2-\mathrm{O} 4^{\mathrm{iii}}$ & $77.35(5)$ \\
\hline $\mathrm{C} 6-\mathrm{C} 5-\mathrm{H} 5 \mathrm{C}$ & 109.5 & $\mathrm{O} 5-\mathrm{Na} 2-\mathrm{O} 4^{\mathrm{iii}}$ & $107.75(5)$ \\
\hline $\mathrm{H} 5 \mathrm{~A}-\mathrm{C} 5-\mathrm{H} 5 \mathrm{C}$ & 109.5 & $\mathrm{O} 6^{\mathrm{v}}-\mathrm{Na} 2-\mathrm{O} 3$ & $103.39(6)$ \\
\hline $\mathrm{H} 5 \mathrm{~B}-\mathrm{C} 5-\mathrm{H} 5 \mathrm{C}$ & 109.5 & $\mathrm{O} 7-\mathrm{Na} 2-\mathrm{O} 3$ & $78.33(5)$ \\
\hline $\mathrm{O} 6-\mathrm{C} 6-\mathrm{O} 5$ & $122.86(17)$ & $\mathrm{O} 5-\mathrm{Na} 2-\mathrm{O} 3$ & $110.60(5)$ \\
\hline $\mathrm{O} 6-\mathrm{C} 6-\mathrm{C} 5$ & $121.13(18)$ & $\mathrm{O} 4^{\mathrm{iii}-\mathrm{Na} 2-\mathrm{O} 3}$ & $138.49(5)$ \\
\hline $\mathrm{O} 5-\mathrm{C} 6-\mathrm{C} 5$ & $116.00(17)$ & $\mathrm{O} 6^{\mathrm{v}}-\mathrm{Na} 2-\mathrm{O} 2^{\mathrm{ii}}$ & $99.73(6)$ \\
\hline $\mathrm{O} 33^{\mathrm{ii}}-\mathrm{Cu} 1-\mathrm{O} 3$ & 180.0 & $\mathrm{O} 7-\mathrm{Na} 2-\mathrm{O} 2^{\mathrm{ii}}$ & $77.30(5)$ \\
\hline $\mathrm{O}^{3 i}-\mathrm{Cu} 1-\mathrm{O} 2^{\mathrm{ii}}$ & $95.78(5)$ & $\mathrm{O} 5-\mathrm{Na} 2-\mathrm{O} 2^{\mathrm{ii}}$ & $171.22(5)$ \\
\hline $\mathrm{O} 3-\mathrm{Cu} 1-\mathrm{O} 2^{\mathrm{ii}}$ & $84.22(5)$ & 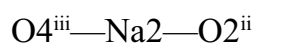 & $81.02(5)$ \\
\hline $\mathrm{O} 3^{\mathrm{ii}}-\mathrm{Cu} 1-\mathrm{O} 2$ & $84.22(5)$ & $\mathrm{O} 3-\mathrm{Na} 2-\mathrm{O} 2^{\mathrm{ii}}$ & $61.06(4)$ \\
\hline $\mathrm{O} 3-\mathrm{Cu} 1-\mathrm{O} 2$ & $95.78(5)$ & $\mathrm{C} 2-\mathrm{O} 2-\mathrm{Cu} 1$ & $112.95(10)$ \\
\hline $\mathrm{O} 2^{\mathrm{ii}}-\mathrm{Cu} 1-\mathrm{O} 2$ & $180.00(6)$ & $\mathrm{C} 2-\mathrm{O} 2-\mathrm{Na} 1^{\mathrm{i}}$ & $137.32(10)$ \\
\hline $\mathrm{O} 3^{\mathrm{ii}}-\mathrm{Cu} 1-\mathrm{Na}^{\mathrm{i}}$ & $99.26(3)$ & $\mathrm{Cu} 1-\mathrm{O} 2-\mathrm{Na}^{\mathrm{i}}$ & $103.76(5)$ \\
\hline $\mathrm{O} 3-\mathrm{Cu} 1-\mathrm{Na} 1^{\mathrm{i}}$ & $80.74(3)$ & $\mathrm{C} 2-\mathrm{O} 2-\mathrm{Na}^{2 \mathrm{ii}}$ & $116.47(9)$ \\
\hline $\mathrm{O} 2^{\mathrm{ii}}-\mathrm{Cu} 1-\mathrm{Na} 1^{\mathrm{i}}$ & $137.87(3)$ & $\mathrm{Cu} 1-\mathrm{O} 2-\mathrm{Na} 2^{\mathrm{ii}}$ & $96.36(5)$ \\
\hline $\mathrm{O} 2-\mathrm{Cu} 1-\mathrm{Na} 1^{\mathrm{i}}$ & $42.13(3)$ & $\mathrm{Na} 1^{\mathrm{i}}-\mathrm{O} 2-\mathrm{Na} 2^{\mathrm{ii}}$ & $78.74(4)$ \\
\hline 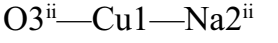 & $44.26(4)$ & $\mathrm{C} 4-\mathrm{O} 3-\mathrm{Cu} 1$ & $128.07(10)$ \\
\hline $\mathrm{O} 3-\mathrm{Cu} 1-\mathrm{Na} 2^{\mathrm{ii}}$ & $135.74(4)$ & $\mathrm{C} 4-\mathrm{O} 3-\mathrm{Na} 2$ & $126.27(10)$ \\
\hline $\mathrm{O} 2^{\mathrm{ii}}-\mathrm{Cu} 1-\mathrm{Na} 2^{\mathrm{ii}}$ & $130.43(4)$ & $\mathrm{Cu} 1-\mathrm{O} 3-\mathrm{Na} 2$ & $102.59(5)$ \\
\hline $\mathrm{O} 2-\mathrm{Cu} 1-\mathrm{Na} 2^{\mathrm{ii}}$ & $49.57(4)$ & $\mathrm{C} 4-\mathrm{O} 4-\mathrm{Na} 1^{\mathrm{i}}$ & $133.95(10)$ \\
\hline $\mathrm{Na} 1^{\mathrm{i}}-\mathrm{Cu} 1-\mathrm{Na} 2^{\mathrm{ii}}$ & $55.23(2)$ & $\mathrm{C} 4-\mathrm{O} 4-\mathrm{Na} 2^{\mathrm{i}}$ & $131.20(11)$ \\
\hline $\mathrm{O} 4^{4 i-}-\mathrm{Na} 1-\mathrm{O} 4^{\mathrm{iii}}$ & 180.0 & $\mathrm{Na} 1^{\mathrm{i}}-\mathrm{O} 4-\mathrm{Na}^{2}{ }^{\mathrm{i}}$ & $84.62(5)$ \\
\hline $\mathrm{O} 4^{\mathrm{ii}-}-\mathrm{Na} 1-\mathrm{O} 2^{\mathrm{ii}}$ & $87.05(5)$ & $\mathrm{C} 6-\mathrm{O} 5-\mathrm{Na} 2$ & $154.01(12)$ \\
\hline 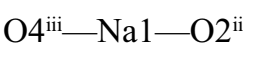 & $92.95(5)$ & $\mathrm{C} 6-\mathrm{O} 5-\mathrm{H} 5$ & 109.4 \\
\hline $\mathrm{O} 4^{\mathrm{ii}}-\mathrm{Na} 1-\mathrm{O} 2^{\mathrm{iii}}$ & $92.95(5)$ & $\mathrm{Na} 2-\mathrm{O} 5-\mathrm{H} 5$ & 94.7 \\
\hline 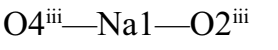 & $87.05(5)$ & $\mathrm{C} 6-\mathrm{O} 6-\mathrm{Na} 2^{\mathrm{v}}$ & $133.48(13)$ \\
\hline 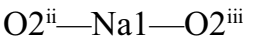 & 180.0 & $\mathrm{Na} 2-\mathrm{O} 7-\mathrm{Na} 1$ & $81.12(5)$ \\
\hline 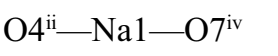 & $80.02(5)$ & $\mathrm{Na} 2-\mathrm{O} 7-\mathrm{H} 7 \mathrm{~B}$ & 117.7 \\
\hline 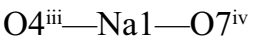 & $99.98(5)$ & $\mathrm{Na} 1-\mathrm{O} 7-\mathrm{H} 7 \mathrm{~B}$ & 119.5 \\
\hline $\mathrm{O} 2^{\mathrm{ii}}-\mathrm{Na} 1-\mathrm{O} 7^{\mathrm{iv}}$ & $97.70(5)$ & $\mathrm{Na} 2-\mathrm{O} 7-\mathrm{H} 7 \mathrm{~A}$ & 120.0 \\
\hline $\mathrm{O} 2^{i \mathrm{ii}-\mathrm{Na} 1-\mathrm{O} 7^{\mathrm{iv}}}$ & $82.30(5)$ & $\mathrm{Na} 1-\mathrm{O} 7-\mathrm{H} 7 \mathrm{~A}$ & 116.7 \\
\hline
\end{tabular}




\section{supporting information}

$\begin{array}{llll}\mathrm{O} 4 & \mathrm{ii}-\mathrm{Na} 1-\mathrm{O} 7 & 99.98(5) & \mathrm{H} 7 \mathrm{~B}-\mathrm{O} 7-\mathrm{H} 7 \mathrm{~A}\end{array}$

$\mathrm{O} 4{ }^{\mathrm{iii}-\mathrm{Na} 1-\mathrm{O} 7}$

$80.02(5)$

Symmetry codes: (i) $x, y+1, z$; (ii) $-x+1,-y+2,-z+1$; (iii) $x, y-1, z$; (iv) $-x+1,-y+1,-z+1$; (v) $-x+1, y,-z+3 / 2$.

Hydrogen-bond geometry $\left(\AA,{ }^{\circ}\right)$

\begin{tabular}{lllll}
\hline$D-\mathrm{H} \cdots A$ & $D-\mathrm{H}$ & $\mathrm{H} \cdots A$ & $D \cdots A$ & $D-\mathrm{H} \cdots A$ \\
\hline $\mathrm{O} 7-\mathrm{H} 7 A \cdots \mathrm{O} 1$ & 0.79 & 2.05 & $2.835(2)$ & 172 \\
$\mathrm{O} 7-\mathrm{H} 7 B \cdots \mathrm{O} 1^{\text {vi }}$ & 0.80 & 2.03 & $2.8104(19)$ & 168 \\
\hline
\end{tabular}

Symmetry code: (vi) $-x+1 / 2,-y+3 / 2,-z+1$. 